DOI 10. 18307/2018. 0507

(c) 2018 by Journal of Lake Sciences

\title{
生态最相关水文指标的优选及其在洞庭湖环境流量估算中的应用”
}

\author{
程俊翔 ${ }^{1,2}$, 徐力刚 ${ }^{1,2,3 * *}$, 姜加虎 ${ }^{1,2}$ \\ (1: 中国科学院南京地理与湖泊研究所,中国科学院流域地理学重点实验室,南京 210008) \\ (2: 中国科学院大学,北京 100049) \\ (3:江西省山江湖开发治理委员会办公室,南昌 330046)
}

\begin{abstract}
摘 要: 水文改变指标 (IHA) 能够较为全面地描述水文状况,在评估水文情势改变及其生态系统影响方面具有广泛的应 用. 尽管该指标体系较为完善,但是数量众多的水文变量仍然存在信息冗余问题. 根据洞庭湖城陵矶水文站 1955-2014 年的径流量数据, 采用主成分分析 (PCA) 篮选了生态最相关水文指标 (ERHIs), 结合 ERHIs 改进了用于估算环境流量的 变化范围法 (RVA), 并将其应用在洞庭湖出口的环境流量估算中. 基于 PCA 选取了年最大 90 日流量、年最小 3 日流量、 年最小流量出现时间、 3 月流量、6 月流量、流量逆转次数和低流量年内平均历时 7 个变量作为洞庭湖出口的 ERHIs. 纵向 和横向的对比分析都表明选取的 ERHIs 是合理的. ERHIs 不仅有效缓解了 IHA 的穴余性问题, 还有利于抓住最关键的生 态水文变量. 根据 ERHIs 改进的 RVA 方法在设定洞庭湖出口环境流量时, 极大地简化原来的众多管理目标, 对生态水文 研究、水资源管理和生态保护都具有重要的参考价值和借鉴意义.
\end{abstract}

关键词: 生态水文;水文改变指标;呪余性;主成分分析;环境流量;洞庭湖

\section{Optimal selection of the most ecologically relevant hydrologic indicators and its application for environmental flow calculation in Lake Dongting}

\author{
CHENG Junxiang ${ }^{1,2}$, XU Ligang ${ }^{1,2,3 * *}$ \& JIANG Jiahu ${ }^{1,2}$ \\ (1: Key Laboratory of Watershed Geographic Sciences, Nanjing Institute of Geography and Limnology, Chinese Academy of \\ Sciences, Nanjing 210008, P.R. China) \\ (2: University of Chinese Academy of Sciences, Beijing 100049, P.R.China) \\ (3: Office of Mountain-River-Lake Development Committee of Jiangxi Province, Nanchang 330046, P.R.China)
}

Abstract: The method of indicators of hydrologic alteration (IHA) provides a relatively comprehensive way to describe the full range of hydrological regimes and is used globally to assess hydrological alteration and its ecosystem influence. This method is relatively sound, while it still has potential redundancy problems caused by a large number of variables in it. Therefore, the principal component analysis (PCA) was used to select the most ecologically relevant hydrologic indicators (ERHIs), based on the flow data at Chenglingji, a standard observation station of Lake Dongting, from 1955 to 2014 . The method of range of variability approach ( RVA) was improved by the selected ERHIs and was used to estimate environmental flow in the outlet of Lake Dongting. Based on the results of PCA, seven ERHIs were selected: annual maximum 90-day flow, annual minimum 3-day flow, Julian date of each annual 1-day minimum, March flow, June flow, number of hydrologic reversals and median duration of low pulses. It was proved that the seven selected ERHIs were reasonable with the comparison to previous studies and the redundancy analysis. ERHIs not only reduce the redundancy of IHA but also help to grasp the key component of eco-hydrological variables. Moreover, the improved RVA method greatly simplified the original management targets in estimating environmental flow in the outlet of Lake Dongting. This stud$\mathrm{y}$ is of great value and significance for eco-hydrological research, water resources management and ecological protection.

* 国家重点研发计划项目(2018YFC0407600)、国家自然科学基金项目 (41771235,41661018)、江西省重点研发计划 项目 (20171BBH80015)、江苏高校水处理技术与材料协同创新中心项目和江西省科学院重点科研 (对外合作) 项 目 (2018-YZD2-03) 联合资助. 2018-01-15 收稿; 2018-03-19 收修改稿. 程俊翔 (1991 ), 男, 博士研究生; E-mail: chengjunxiang15@163.com.

** 通信作者;E-mail: lgxu@ niglas.ac.cn. 
Keywords: Eco-hydrology; indicators of hydrologic alteration; redundancy; principal component analysis; environmental flow; Lake Dongting

生态水文过程是研究水文过程与生态过程之间功能关系的科学, 而水文情势变化决定了水域生态系统的 结构和功能 ${ }^{[1-2]}$. 定量描述水文变量与生态变量之间的关系是开展生态水文学研究的基础,也是国内外研究的 热点问题之一 ${ }^{[3-4]}$. 早期研究往往只考虑单一水文变量, 无论是对水文情势的整体变化特征, 还是对生态系统 整体性的认识都不充分 ${ }^{[5]}$. 随着研究的深人, Richter 等 ${ }^{[6]}$ 提出的水文改变指标 (indicators of hydrologic alteration,IHA) 不仅能够充分描述径流量或水位年际和年内的全局变化特征, 还建立了不同的水文变量与水 生生境、栖息地环境等生态特征的联系 ${ }^{[7]}$. 为了进一步明确适宜的环境流量, 基于 IHA 的变化范围法 ( range of variability approach, RVA) 也迅速发展起来, 并可用于定量评估水文改变度和估算生态环境需水量 ${ }^{[8]}$. IHA 包 含了 33 个水文变量, 是目前公认的相对最全面、最系统的一套指标体系. Suen ${ }^{[9]}$ 采用 IHA 和 RVA 评估了气候 变化下的水文情势改变, 指出水文情势改变严重影响了当地的淡水生态系统. Zhang 等 ${ }^{[10]}$ 基于 IHA、RVA 和环 境流量成分 (environmental flow components, EFCs) 等方法, 分析了珠江流域 3 个大型水库对东江水文情势和环 境流量的影响,研究结果表明水库对东江的水文情势产生了严重影响, 且 EFCs 可以为流域的环境需水量提供 决策依据. 此外, IHA 体系还可以用于评估水文情势在空间上的变化. Jiang 等 ${ }^{[11]}$ 根据宜昌、汉口、大通等长江 干流 7 个水文站的径流量数据, 采用 RVA 分析了三峡大坝对长江中下游水情的影响, 认为三峡水库蓄水对长 江径流的变化率和年极端流量的影响较大, 且影响程度随着大坝距离的增加而减弱. 目前, IHA 体系在水文情 势改变评估、生态影响评估、环境流量估算等方面都有着重要的地位和广泛的应用 ${ }^{[12-14]}$.

Olden 等 ${ }^{[15]}$ 对已公开发表文献中的 171 个水文变量的分析表明,众多水文变量之间的信息冗余性问题 十分突出, 而 33 个 IHA 基本上能覆盖大多数变量的信息. 尽管 IHA 体系相对完善, 但是 33 个水文变量仍然 较多,在水资源管理实践中也难以操作和管理 ${ }^{[16]}$. 此外, IHA 变量之间也存在着自相关性和信息冗余性的问 题 ${ }^{[17]}$. Yang 等 ${ }^{[18]}$ 综合采用遗传规划算法 (genetic programming, GP)、主成分分析 (principal component analysis, PCA) 和个体生态学矩阵 (autecology matrix, AM) 3 种方法, 从 IHA 中选取了年最小流量出现时间、 上升率、流量逆转次数、年最大 3 日流量、年最小 7 日流量和 5 月流量这 6 个变量作为 Illinois 河的生态最相 关水文指标 (the most ecologically relevant hydrologic indicators, ERHIs). Gao 等 ${ }^{[19]}$ 通过多元线性回归分析了 IHA 变量的多重共线性问题, 并基于 PCA 分别篮选了模拟数据集和实测数据集的 ERHIs, 结果表明不同数 据集的分析结果存在一定的差异性. 一般地, ERHIs 就是通过较少的几个关键水文变量来代表众多的水文 变量,并可以反映出最重要的生态相关信息 ${ }^{[18]}$. 其优点是能有效减少众多水文变量的信息几余性,并尽可 能多地保留原数据的关键信息. 考虑到 IHA 变量有 33 个之多, 那么如何通过代表性的几个水文变量有效地 表征生态水文信息仍然是目前研究所面临的巨大挑战,特别是关于 ERHIs 的选取, 已经成为构建生态水文 联系的关键问题 ${ }^{[20]}$.

因此, 本文以洞庭湖城陵矶水文站的逐日径流量为数据基础, 以 IHA 变量的咒余性为研究背景, 采用 PCA 方法从 IHA 变量中篮选洞庭湖的 ERHIs, 作为联系水文与生态的重要纽带. 然后, 分别从纵向和横向两 个层面讨论 ERHIs 的合理性和优越性, 并重点讨论 ERHIs 的生态水文响应关系. 最后, 基于优选出的 ERHIs, 改进用于估算环境流量的 RVA 方法, 并将其应用于设定适宜的洞庭湖出口环境流量, 期望为洞庭湖 的水资源管理和生态保护提供一定的科学参考.

\section{1 材料与方法}

\section{1 数据}

洞庭湖主要接纳长江三口 (松滋、太平和藕池) 分流和湖南四水 (湘江、资水、沅江和澧水) 来水, 经过湖 泊调蓄后, 通过唯一的出口——城陵矶再次排泄人江. 水文数据为洞庭湖出口城陵矶水文站的逐日流量 (单 位: $\mathrm{m}^{3} / \mathrm{s}$ ), 时间序列为 1955-2014 年, 该数据集来源于湖南省水文水资源勘测局. 洞庭湖出口的多年平均 径流量为 $8779 \mathrm{~m}^{3} / \mathrm{s}$, 变异系数为 0.74 .

\section{2 研究方法}

1.2.1 水文改变指标 (IHA) IHA 包含了 5 组 33 个水文变量, 涵盖了径流量的量级、历时、出现时间、频率、 
变化率等全部水文特征. 由于研究时段内洞庭湖出口未出现断流现象, 故本研究不考虑 “断流天数”这一变 量,其余 32 个水文变量如表 1 所示. 基于 IHA 7.1 软件,采用非参数化方法计算洞庭湖出口径流量的 32 个 水文变量. 非参数化方法计算得到的数值为各水文变量的中值和分位数, 也更加适用于非正态分布的水文 数据 ${ }^{[7]}$. 各水文变量的详细说明和计算方法请参见 IHA 软件使用手册 ${ }^{[7]}$.

表 1 IHA 的分组及变量

Tab. 1 Groups and variables of IHA

\begin{tabular}{|c|c|c|}
\hline IHA 分组 & 水文变量(单位) & 说明 \\
\hline 第 1 组: 月流量 & 各月流量 $\left(\mathrm{m}^{3} / \mathrm{s}\right)$ & $1-12$ 月的各月流量 \\
\hline $\begin{array}{l}\text { 第 } 2 \text { 组: 年极端流量 } \\
\text { 及历时 }\end{array}$ & $\begin{array}{l}\text { 年最小 } 1 \text { 日流量 }\left(\mathrm{m}^{3} / \mathrm{s}\right) \\
\text { 年最小 } 3 \text { 日流量 }\left(\mathrm{m}^{3} / \mathrm{s}\right) \\
\text { 年最小 } 7 \text { 日流量 }\left(\mathrm{m}^{3} / \mathrm{s}\right) \\
\text { 年最小 } 30 \text { 日流量 }\left(\mathrm{m}^{3} / \mathrm{s}\right) \\
\text { 年最小 } 90 \text { 日流量 }\left(\mathrm{m}^{3} / \mathrm{s}\right) \\
\text { 年最大 } 1 \text { 日流量 }\left(\mathrm{m}^{3} / \mathrm{s}\right) \\
\text { 年最大 } 3 \text { 日流量 }\left(\mathrm{m}^{3} / \mathrm{s}\right) \\
\text { 年最大 } 7 \text { 日流量 }\left(\mathrm{m}^{3} / \mathrm{s}\right) \\
\text { 年最大 } 30 \text { 日流量 }\left(\mathrm{m}^{3} / \mathrm{s}\right) \\
\text { 年最大 } 90 \text { 日流量 }\left(\mathrm{m}^{3} / \mathrm{s}\right) \\
\text { 基流指数 }\end{array}$ & $\begin{array}{l}3 \text { 日、7 日、30日、90日流量指某水文年内的多日滑动平均 } \\
\text { 流量,年最大(或最小) 多日流量为该水文年的相应多日滑 } \\
\text { 动平均流量的最大值 (或最小值); 基流指数定义为某水文 } \\
\text { 年的最小 } 7 \text { 日滑动平均流量与该水文年平均流量的比值 }\end{array}$ \\
\hline $\begin{array}{l}\text { 第 } 3 \text { 组:年极端流量 } \\
\text { 出现时间 }\end{array}$ & $\begin{array}{l}\text { 年最小流量出现时间 (儒略日) } \\
\text { 年最大流量出现时间 (儒略日) }\end{array}$ & $\begin{array}{l}\text { 若某水文年内有多日出现最大 (或最小) 流量, 仅记录其最 } \\
\text { 早出现的时间 }\end{array}$ \\
\hline $\begin{array}{l}\text { 第 } 4 \text { 组:高低流量发 } \\
\text { 生频率和历时 }\end{array}$ & $\begin{array}{l}\text { 低流量年内发生次数( 次) } \\
\text { 低流量年内平均历时 }(\mathrm{d}) \\
\text { 高流量年内发生次数(次) } \\
\text { 高流量年内平均历时 }(\mathrm{d})\end{array}$ & $\begin{array}{l}\text { 在某水文年内, 高、低流量的阈值设定为 } 50 \% \text { 分位数 } \pm 25 \% \text {, } \\
\text { 即超过 } 75 \% \text { 分位数的划分为高流量, 低于 } 25 \% \text { 分位数的划 } \\
\text { 分为低流量 }\end{array}$ \\
\hline $\begin{array}{l}\text { 第 } 5 \text { 组: 流量改变率 } \\
\text { 和频率 }\end{array}$ & $\begin{array}{l}\text { 上升率 }\left(\mathrm{m}^{3} /(\mathrm{s} \cdot \mathrm{d})\right) \\
\text { 下降率 }\left(\mathrm{m}^{3} /(\mathrm{s} \cdot \mathrm{d})\right) \\
\text { 流量逆转次数 }\end{array}$ & $\begin{array}{l}\text { 上升率 (下降率) 为某水文年内连续两日流量的正 (负) 变 } \\
\text { 化率; 流量逆转次数指某水文年内日流量由增加变为减少 } \\
\text { 或由减少变为增加的次数 }\end{array}$ \\
\hline
\end{tabular}

1.2.2 主成分分析 (PCA) PCA 是通过降维的思想把大量相关变量转化为少数几个不相关变量, 并尽可能 保留原始数据的信息, 在水文学、生态学等领域具有重要的应用价值 ${ }^{[21-22]}$. 在分析之前, 首先对数据进行标 准化处理, 以消除量纲不同和数值大小的影响. 主成分 ( PC) 的个数 $k$ 的选取主要基于以下两个原则: (1) 特 征值 $\geqslant 1 ;$; (2) 累积贡献率达到 $70 \% \sim 90 \%{ }^{[23-24]}$. 然后, 根据所确定的 $k$ 个主成分的因子载荷矩阵, 选取载荷 值 (绝对值) 最大的变量作为该主成分的解释变量. PCA 的基本原理和详细步骤请参阅文献 [24].

1.2.3 环境流量估算方法 根据 IHA 的参数化计算结果, 分别得到 32 个水文变量的平均值和标准差. RVA 通过为每一个水文变量划分一个阈值范围来设定环境流量的管理目标, 而这些变量的阈值一般是根据该变 量的“平均值沶准差”计算得来的 ${ }^{[7-8]}$. 考虑到 32 个水文变量的复杂性和尣余性, Smakhtin 等 ${ }^{[17]}$ 建议保留 12 个月份的流量以反映流量在季节上的变化特征, 以及最大和最小 1 日、30日流量来反映极端流量的变化 特征, 即将原来的 32 个变量压缩到 16 个. 洞庭湖出口的多年月平均径流量波动较大, 具有明显的季节差异 性, 最大值与最小值相差高达 $14473 \mathrm{~m}^{3} / \mathrm{s}$. 因此, 在设定洞庭湖出口的环境流量目标时, 有必要保留 12 个月 的流量. 在此基础上, 增加通过 PCA 篎选出的 ERHIs, 一起作为洞庭湖出口环境流量的管理目标, 以改进原 来的 RVA 方法. 这样既可以减少管理目标的数量, 同时也有效地保留了 IHA 的关键信息.

\section{2 结果与讨论}

\section{$2.1 \mathrm{IHA}$ 的圥余性分析}

通过相关性分析, 可以初步确定 32 个 IHA 之间的相关性, 图 1 展示了各变量之间的相关系数. 为了便 
于比较,这里根据相关系数的绝对值来进行分析. 从图 1 可以看出, 部分水文变量之间存在着较强的相关 性, 其相关系数甚至超过了 0.90. 年最小 1 日、3 日、7 日、30日和 90 日流量之间的相关性较强, 平均值为 0.71 ; 年最大 1 日、 3 日、 7 日、30日和 90 日流量、基流指数之间的相关性更强,平均值为 0.79 . 此外,枯水期 (12月一次年 3 月) 流量之间也具有较强的相关性; 丰水期流量 (7-9 月) 除自身外, 还与年最大 1 日、3 日、7 日、30 日、90 日流量、基流指数、低流量年内发生次数和历时、上升率和下降率等参数之间也存在着较强的 相关关系; 年最大 1 日、3 日、7 日、30日、90日流量以及基流指数与低流量年内发生次数和历时、上升率和下 降率之间也具有较强的相关性. 由此可见, 32 个 IHA 变量之间存在着高度自相关性,即信息冗余问题比较 突出.

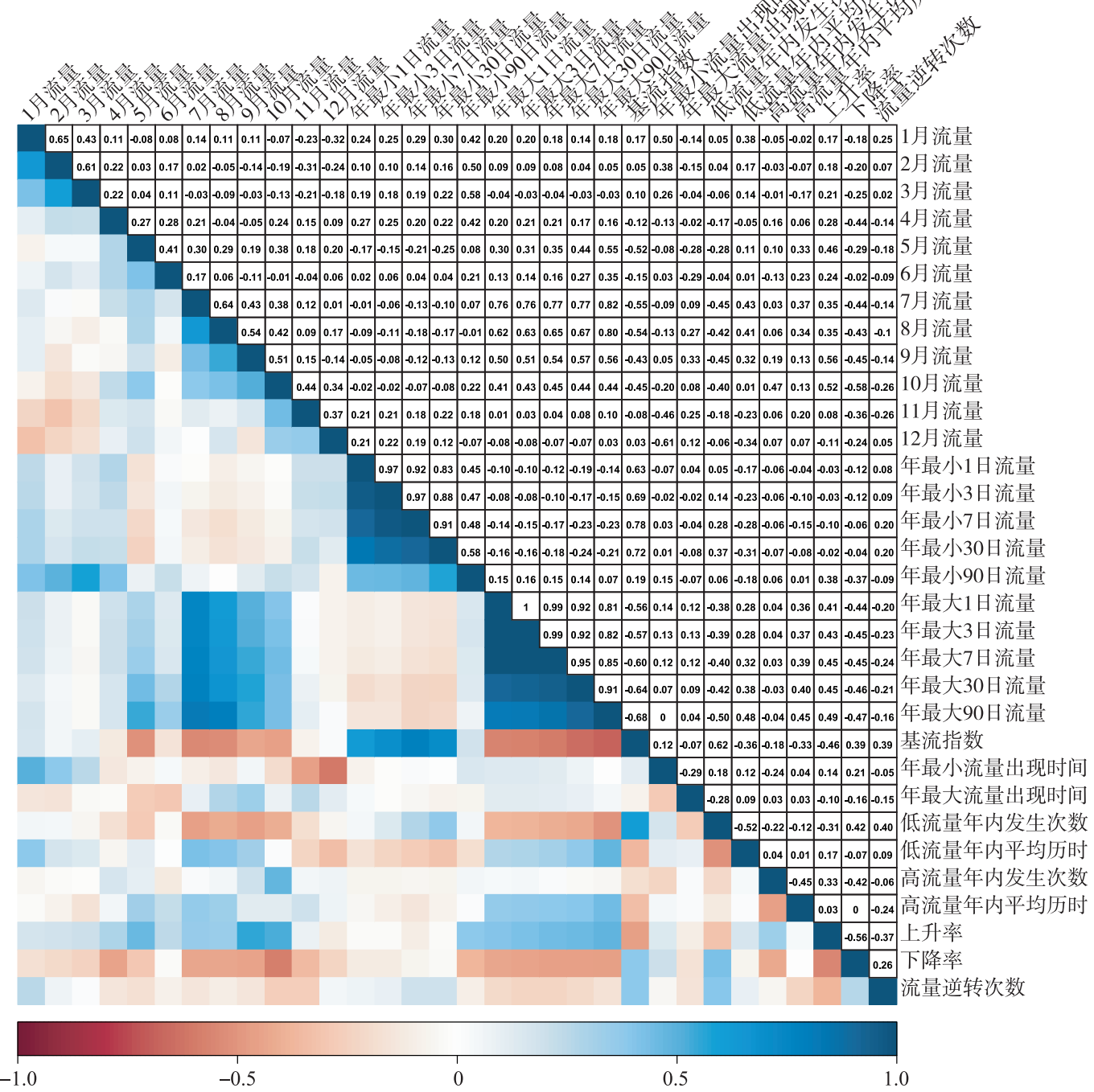

图 132 个 IHA 的相关关系

Fig.1 Correlations among the thirty-two IHAs

\subsection{ERHIs 的选取}

鉴于 IHA 变量之间的冗余性问题比较突出, ERHIs 的选取就显得尤为重要. 基于 PCA 的 32 个水文变量 的特征值和累积贡献率如图 2 所示. 第 1 主成分 (PC1) 的特征值为 8.98 , 贡献率为 $28 \%$; 第 2 主成分 (PC2) 
的特征值为 4.88, PC1 和 PC2 的累积贡献率为 $43 \%$. 前 8 个主成分的特征值都大于 1 , 且累积贡献率约为 $80 \%$. 根据主成分的提取原则, PC1 PC8 即为所需的主成分.

为了进一步确定各主成分的解释变量, 表 2 列出了 PC1 PC8 的因子载荷矩阵. 为方便比较, 这里以载荷 值的绝对值来进行比较分析. 对 PC1 而言, 年最大 1 日、3 日、7 日、30日、90日流量和基流指数的载荷值都较 高, 其中最高载荷值为年最大 90 日流量. 而 PC2 的载荷值较高的变量有年最小 1 日、 3 日、 7 日、30 日和 90 日 流量, 其中年最小 3 日流量的载荷值最高. 后面 6 个主成分 $(\mathrm{PC} 3 \sim \mathrm{PC} 8)$ 的高载荷值相对较少, 且分布比较分 散. PC3 PC8 的最高载荷值对应的变量分别为年最小流量出现时间、 3 月流量、 6 月流量、流量逆转次数、 3 月 流量和低流量年内平均历时. 其中,PC4 和 PC7 的最高载荷值所对应的变量均为 3 月流量. 因此, 最终选取的 前 8 个主成分 $(\mathrm{PC} 1 \sim \mathrm{PC} 8)$ 的解释变量共有 7 个, 依次为年最大 90 日流量、年最小 3 日流量、年最小流量出现 时间、3 月流量、6月流量、流量逆转次数和低流量年内平均历时, 也即洞庭湖出口的 7 个 ERHIs.

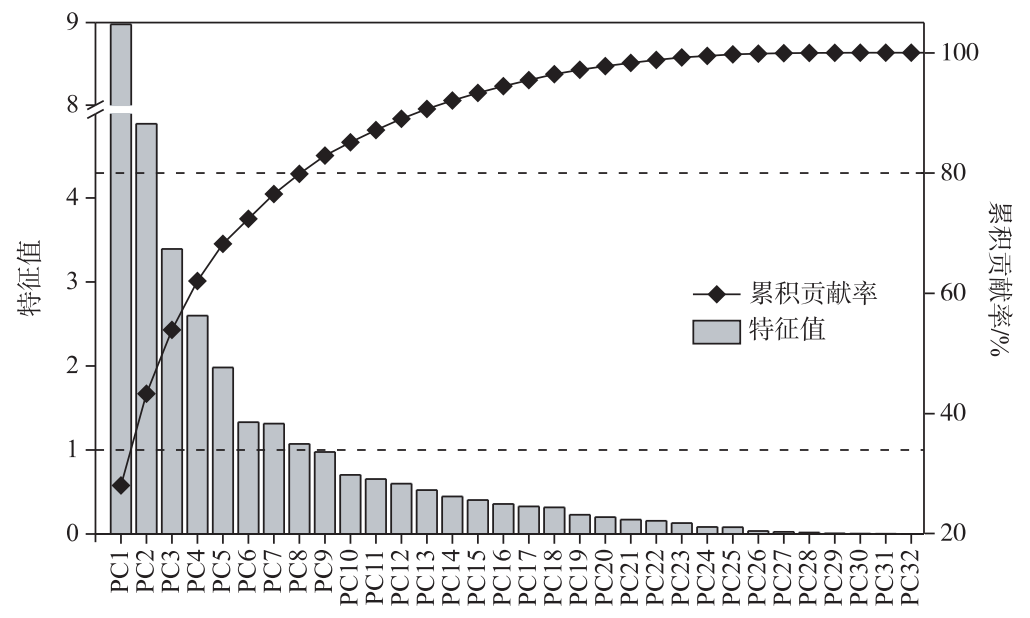

图 2 基于主成分分析的特征值和累积贡献率的变化

Fig. 2 Variations of eigenvalue and cumulative contribution rate explained based on PCA

\subsection{ERHIs 的合理性分析}

IHA 变量之间的自相关性和冗余性问题正受到越来越多的关注. Turner 等 ${ }^{[25]}$ 在研究径流改变和与生态 显著相关的水力环境的改变时, 揭示了多径流变量之间的冗余性问题. Smakhtin 等 ${ }^{[17]}$ 在利用 IHA 变量估算 环境流量时, 指出了年最小多日流量之间的差异小于 $6 \%$, 存在着较高的自相关性, 且年最大多日流量表现 出了同样的特征. 为了有效地缓解众多水文变量的穴余性问题, 部分学者也开展了相应的研究. 基于 33 个 IHA 变量, 相关文献中 ERHIs 的选取结果如表 3 所示. 其中, Gao 等 ${ }^{[19]}$ 根据 PCA 研究了不同数据集的 ERHIs : 模拟数据集确定了 4 个 ERHIs; 实测数据集则选取了 8 个 ERHIs (表 3 ). 这也充分表明不同数据集之 间的分析结果可能存在一定的差异.

与以上研究成果相比, 本文确定的 7 个 ERHIs 分别为年最大 90 日流量、年最小 3 日流量、年最小流量 出现时间、 3 月流量、 6 月流量、流量逆转次数和低流量年内平均历时, 在数量上和具体的变量上都与以上研 究结果不完全相同. 这种差异性很可能是由于不同的研究对象表现出不同的水文特征所导致的,但是这些 ERHIs 之间仍然存在着一些共性. 从表 3 可以看出, 所有研究结果中都包含了一个年最大多日流量和一个 年最小多日流量, 而这些变量也正是前两个 PC 的高载荷值发生聚类的地方 (表 2). 其次, 年最小流量出现 时间在各研究结果中也都出现了 (除 Gao 等 ${ }^{[19]}$ 的研究外). 而年极端流量的出现时间恰恰也是水生生物进 人生命循环周期的重要信号 ${ }^{[26]}$, 在实际研究中需要格外关注. 另外, 月流量、高低流量发生次数和历时以及 变化率等组别基本上也都包含了至少一个变量. 也就是说, 洞庭湖出口的 ERHIs 基本上涉及到了 IHA 的所 有 5 个分组 (表 1), 且含有每组中的至少一个变量. 这也充分说明, 根据 PCA 篎选出的 ERHIs 在减少 32 个 IHA 变量冗余性的同时,有效地保留了原始数据的信息. 
表 2 前 8 个主成分的载荷值"

Tab.2 Loadings for the first eight principal components

\begin{tabular}{|c|c|c|c|c|c|c|c|c|}
\hline IHA 变量 & PC1 & $\mathrm{PC} 2$ & PC3 & PC4 & PC5 & PC6 & PC7 & PC8 \\
\hline 1 月流量 & 0.07 & 0.46 & 0.66 & -0.20 & -0.21 & 0.09 & 0.11 & 0.08 \\
\hline 2 月流量 & 0.04 & 0.38 & 0.43 & -0.51 & 0.04 & -0.10 & 0.36 & 0.22 \\
\hline 3 月流量 & 0.02 & 0.41 & 0.17 & -0.61 & 0.00 & -0.19 & $\underline{0.39}$ & 0.07 \\
\hline 4 月流量 & 0.22 & 0.43 & -0.15 & -0.29 & 0.23 & 0.14 & -0.02 & -0.28 \\
\hline 5 月流量 & 0.52 & 0.02 & -0.10 & -0.10 & 0.50 & 0.25 & -0.04 & -0.18 \\
\hline 6 月流量 & 0.22 & 0.18 & 0.11 & -0.14 & 0.67 & 0.24 & 0.08 & -0.20 \\
\hline 7 月流量 & 0.78 & 0.12 & 0.14 & 0.25 & -0.04 & 0.18 & 0.05 & -0.09 \\
\hline 8 月流量 & 0.75 & 0.05 & -0.01 & 0.27 & -0.18 & 0.10 & 0.20 & 0.13 \\
\hline 9 月流量 & 0.65 & 0.08 & -0.10 & -0.03 & -0.42 & -0.14 & -0.14 & 0.05 \\
\hline 10 月流量 & 0.58 & 0.14 & -0.46 & -0.12 & -0.16 & 0.13 & -0.32 & 0.09 \\
\hline 11 月流量 & 0.19 & 0.23 & -0.70 & 0.04 & 0.08 & -0.18 & 0.07 & 0.08 \\
\hline 12 月流量 & 0.06 & 0.18 & -0.68 & 0.12 & 0.16 & 0.16 & 0.34 & 0.34 \\
\hline 年最小 1 日流量 & -0.25 & 0.84 & -0.09 & 0.30 & -0.08 & 0.02 & 0.04 & -0.24 \\
\hline 年最小 3 日流量 & -0.28 & $\underline{0.87}$ & -0.06 & 0.30 & -0.05 & 0.04 & -0.05 & -0.17 \\
\hline 年最小 7 日流量 & -0.37 & 0.86 & -0.01 & 0.28 & -0.06 & 0.07 & -0.08 & -0.07 \\
\hline 年最小 30 日流量 & -0.37 & 0.84 & 0.02 & 0.23 & -0.02 & 0.04 & -0.11 & -0.01 \\
\hline 年最小 90 日流量 & 0.13 & 0.76 & -0.02 & -0.39 & 0.10 & -0.22 & 0.01 & 0.19 \\
\hline 年最大 1 日流量 & 0.86 & 0.16 & 0.22 & 0.21 & -0.02 & -0.05 & -0.12 & 0.17 \\
\hline 年最大 3 日流量 & 0.87 & 0.16 & 0.21 & 0.20 & -0.01 & -0.06 & -0.12 & 0.15 \\
\hline 年最大 7 日流量 & 0.89 & 0.14 & 0.20 & 0.20 & 0.00 & -0.04 & -0.12 & 0.13 \\
\hline 年最大 30 日流量 & 0.90 & 0.07 & 0.23 & 0.20 & 0.05 & 0.04 & -0.09 & 0.05 \\
\hline 年最大 90 日流量 & $\underline{0.91}$ & 0.08 & 0.17 & 0.20 & 0.10 & 0.17 & 0.08 & -0.03 \\
\hline 基流指数 & -0.82 & 0.44 & 0.12 & 0.23 & -0.10 & 0.03 & -0.08 & 0.02 \\
\hline 年最小流量出现时间 & -0.05 & 0.05 & $\underline{0.76}$ & -0.19 & 0.02 & -0.28 & -0.35 & 0.01 \\
\hline 年最大流量出现时间 & 0.13 & -0.02 & -0.16 & 0.39 & -0.46 & -0.38 & 0.28 & -0.13 \\
\hline 低流量年内发生次数 & -0.63 & 0.03 & 0.27 & 0.15 & 0.19 & 0.12 & -0.35 & 0.37 \\
\hline 低流量年内平均历时 & 0.38 & -0.18 & 0.47 & -0.02 & -0.37 & 0.27 & 0.30 & -0.42 \\
\hline 高流量年内发生次数 & 0.16 & 0.02 & -0.41 & -0.50 & -0.41 & 0.33 & -0.28 & -0.03 \\
\hline 高流量年内平均历时 & 0.42 & 0.01 & 0.05 & 0.41 & 0.50 & -0.30 & 0.15 & 0.06 \\
\hline 上升率 & 0.61 & 0.27 & -0.07 & -0.41 & 0.01 & -0.05 & -0.28 & -0.12 \\
\hline 下降率 & -0.59 & -0.39 & 0.37 & 0.34 & 0.17 & 0.00 & -0.06 & -0.09 \\
\hline 流量逆转次数 & -0.35 & 0.04 & 0.25 & 0.12 & -0.18 & $\underline{0.63}$ & 0.19 & 0.38 \\
\hline
\end{tabular}

* 粗体数值是该主成分中较高的几个载荷值, 带下划线的数值为该主成分的最高载荷值.

表 3 相关文献中基于 IHA 变量选取的 ERHIs

Tab.3 ERHIs selected from IHA variables in some related literatures

\begin{tabular}{|c|c|c|}
\hline 方法 & ERHIs & 参考文献 \\
\hline GP、PCA、AM & $\begin{array}{l}\text { 年最小流量出现时间、上升率、流量逆转次数、年最大 } 3 \text { 日流量、年最小 } 7 \text { 日 } \\
\text { 流量、5月流量 }\end{array}$ & Yang 等 $[18]$ \\
\hline PCA & $\begin{array}{l}\text { 模拟数据集: } 5 \text { 月流量、年 } 30 \text { 日最小流量、上升率、年最大流量出现时间 } \\
\text { 实测数据集:年最小 } 30 \text { 日流量、年最大 } 7 \text { 日流量、 } 2 \text { 月流量、 } 11 \text { 月流量、 } 6 \text { 月 } \\
\text { 流量、 } 3 \text { 月流量、上升率、高流量年内平均历时 }\end{array}$ & Gao 等 ${ }^{[19]}$ \\
\hline PCA & $\begin{array}{l}\text { 年最小 } 7 \text { 日流量、年最大 } 7 \text { 日流量、年最小流量出现时间、年最大流量出现时 } \\
\text { 间、高流量年内发生次数 }\end{array}$ & Zhang 等 ${ }^{[27]}$ \\
\hline PCA & $\begin{array}{l}\text { 年最大 } 90 \text { 日流量、年最小 } 3 \text { 日流量、年最小流量出现时间、 } 3 \text { 月流量、 } 6 \text { 月流 } \\
\text { 量、流量逆转次数、低流量年内平均历时 }\end{array}$ & 本研究 \\
\hline
\end{tabular}


为了进一步分析 ERHIs 的合理性, 图 3 展示了 洞庭湖出口的 ERHIs 之间的相关系数, 显然 7 个 ERHIs 之间的相关性大大减小. 从图 3 可以看出, 相关性最强的为年最大 90 日流量和低流量年内平 均历时, 但是其相关系数仅有 0.48 . 绝大多数 ERHIs 之间的相关系数 (绝对值) 都不超过 0.20 , 甚 至有超过一半以上变量的相关系数 (绝对值) 都小 于 0.10 .

\section{4 洞庭湖出口环境流量的估算}

在洞庭湖的水资源管理实践中,管理人员需要 重点关注洞庭湖出口的 7 个 ERHIs. 由于管理目标 相对较少, ERHIs 在实际管理过程中也更加容易实 现. 例如,在利用 RVA 设定洞庭湖出口的适宜环境 流量时,应当重点关注 ERHIs. 所谓的适宜环境流 量应当是一个流量变化范围, 存在一个流量上限和 下限. 无论是超过流量上限还是小于流量下限都会 在一定程度上对生态系统造成损害, 若长时间维持

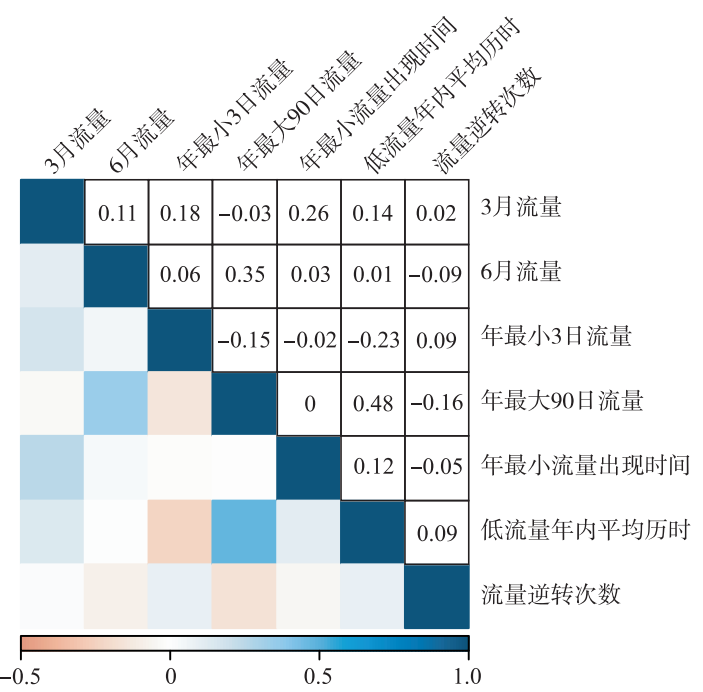

图 37 个 ERHIs 之间的相关关系

Fig. 3 Correlations among the seven ERHIs 在这种状态下, 甚至会对生态系统造成不可逆的损 伤, 只有维持在上下限的范围内才是适宜生态系统的环境流量 ${ }^{[8,28]}$.

根据上面的分析可知,洞庭湖出口的 7 个 ERHIs 不仅保留了 IHA 的有效信息,而且具有十分重要的生 态信息. 所以将 ERHIs 也作为洞庭湖出口环境流量的管理目标, 加上 12 个月的流量 (除去 3 月流量和 6 月 流量两个重复的变量),一共有 17 个水文变量是最终的管理目标 (表 4). 表 4 中的最低阈值和最高阈值分 别对应着最小环境流量和最大环境流量, 也即维持洞庭湖出口生态功能正常所需的最小流量和最大流量. 从生态环境需水的角度来看,低于最小流量或超过最大流量都会给生态系统造成严重的影响.

表 4 基于改进的 RVA 估算的洞庭湖出口环境流量阈值 ${ }^{*}$

Tab.4 Environmental flow of the outlet of Lake Dongting calculated by improved RVA

\begin{tabular}{cccccc}
\hline 水文变量 & 单位 & 平均值 $(\mathrm{m})$ & 标准差 $(\mathrm{sd})$ & 最低阈值 $(\mathrm{m}-\mathrm{sd})$ & 最高阈值 $(\mathrm{m}+\mathrm{sd})$ \\
\hline 1 月流量 & $\mathrm{m}^{3} / \mathrm{s}$ & 2636.85 & 1342.99 & 1293.86 & 3979.84 \\
2 月流量 & $\mathrm{m}^{3} / \mathrm{s}$ & 3476.78 & 1559.54 & 1917.24 & 5036.32 \\
3 月流量 & $\mathrm{m}^{3} / \mathrm{s}$ & 5402.85 & 2238.23 & 3164.62 & 7641.08 \\
4 月流量 & $\mathrm{m}^{3} / \mathrm{s}$ & 8653.25 & 2677.27 & 5975.98 & 11330.52 \\
5 月流量 & $\mathrm{m}^{3} / \mathrm{s}$ & 12568.67 & 4506.08 & 8062.58 & 17074.75 \\
6月流量 & $\mathrm{m}^{3} / \mathrm{s}$ & 14023.57 & 3485.66 & 10537.91 & 17509.23 \\
7 月流量 & $\mathrm{m}^{3} / \mathrm{s}$ & 17114.33 & 5335.71 & 11778.62 & 22450.05 \\
8 月流量 & $\mathrm{m}^{3} / \mathrm{s}$ & 13568.72 & 4933.89 & 8634.83 & 18502.61 \\
9 月流量 & $\mathrm{m}^{3} / \mathrm{s}$ & 11289.48 & 4688.54 & 6600.95 & 15978.02 \\
10 月流量 & $\mathrm{m}^{3} / \mathrm{s}$ & 7985.98 & 3097.10 & 4888.89 & 11083.08 \\
11 月流量 & $\mathrm{m}^{3} / \mathrm{s}$ & 5284.37 & 2314.86 & 2969.51 & 7599.23 \\
12 月流量 & $\mathrm{m}^{3} / \mathrm{s}$ & 3018.76 & 1403.35 & 1615.41 & 4422.12 \\
年最大 90 日流量 & $\mathrm{m}^{3} / \mathrm{s}$ & 16197.90 & 3633.11 & 12564.79 & 19831.01 \\
年最小 3 日流量 & $\mathrm{m}^{3} / \mathrm{s}$ & 1470.79 & 347.65 & 1123.13 & 1818.44 \\
年最小流量出现时间 & 儒略日 & 164.65 & 164.38 & 329.03 & 0.27 \\
流量逆转次数 & 次 & 51.43 & 11.82 & 39.62 & 63.25 \\
低流量年内平均历时 & $\mathrm{d}$ & 26.87 & 23.38 & 3.49 & 50.26 \\
\hline
\end{tabular}

* 年最小流量出现时间的单位为儒略日, 即公历的天数, 其数值大小无实际意义. 由于洞庭湖出口的年最低流量一般出 现在 12 月或次年 1 月, 同时为了保证时间的连续性, 故以当年的年末 (第 329.03 天) 作为最低阈值, 而以次年的年初 (第 0.27 天) 作为最高阈值, 即年最小流量出现时间的变化范围为第 329.03 天至次年的第 0.27 天. 
相比于 32 个变量的 RVA, 根据 ERHIs 改进的 RVA 所设定的洞庭湖出口环境流量的管理目标数量大大 减少, 其在实际管理和操作中的优势显而易见. 此外, 流域水库的调度模式也可以参考环境流量的变化范 围, 从而维持当地生态系统处于正常状态, 这对其他河流、湖泊的水资源管理、生态恢复和保护具有一定的 借鉴意义.

\section{$2.5 \mathrm{ERHIs}$ 的生态水文响应关系}

虽然 IHA 十分全面地描述了洞庭湖出口的水文情势特征, 但是 ERHIs 的选取既减少了 IHA 的自相关 性和冗余性问题, 也有利于进一步篮选出与生态最相关的水文变量. 从洞庭湖出口 7 个 ERHIs 的时间变化 特征来看, 6 月流量和年最大 90 日流量都呈下降趋势, 而 3 月流量、年最小 3 日流量和流量逆转次数具有上 升趋势. 其中, 年最大 90 日流量以每年 $86 \mathrm{~m}^{3} / \mathrm{s}$ 的速率下降, 年最小 3 日流量以每年 $8 \mathrm{~m}^{3} / \mathrm{s}$ 的速率上升, 年 最小流量出现时间有提前的迹象, 而流量逆转次数自 2009 年以后急剧上升 (图 4). 这种变化特征表明了洞 庭湖出口的洪峰流量被大大削弱, 且低流量有所增加. 究其原因, 很可能与流域上众多的水库采用 “蓄洪补 枯” 的运行调度模式有关 ${ }^{[29-30]}$. 据 2009 年的统计数据, 湖南省的水库数量超过 1 万座, 总库容超过 392 亿 $\mathrm{m}^{3}$,约占 $1951-2009$ 年多年平均径流量的 $24 \%{ }^{[31]}$.
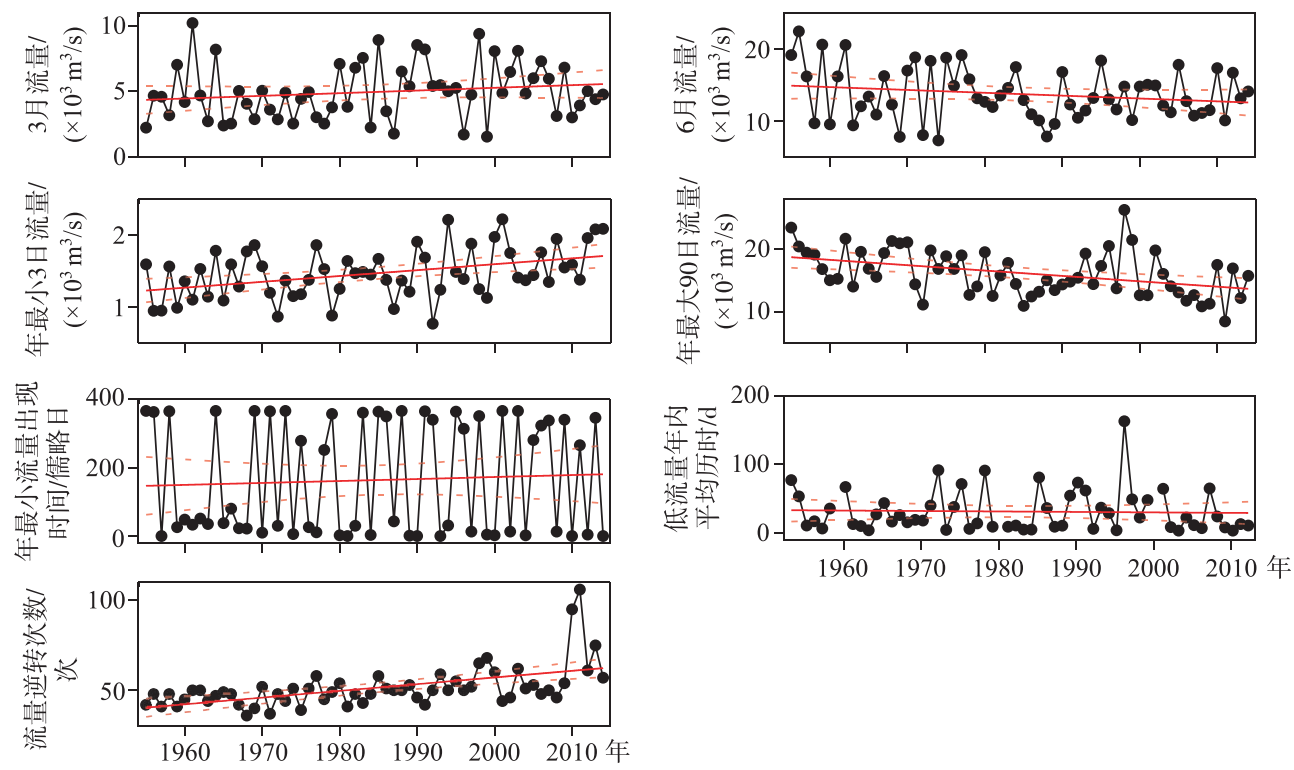

图 4 ERHIs 的时间 (1955-2014 年)变化特征

（红色实线表示线性趋势,两条红色虚线之间区域表示 $95 \%$ 的置信区间）

Fig.4 Temporal variations of ERHIs (1955-2014) (The red lines denote the trend of each ERHIs, the regions between two red dashed lines denote the $95 \%$ confidence interval)

在气候变化和人类活动的双重影响下, 洞庭湖的水文情势发生了显著变化, 并引发了一系列的连锁生 态效应 ${ }^{[32]}$. 由于受到三峡水库和洞庭湖流域上众多水库的共同影响, 洞庭湖的枯水期提前, 导致苔草 (Carex) 群落挤占了曧草 (Phalaris) 群落的生存空间, 促使曧草群落的生长带向高程更低的泥滩地移动 ${ }^{[33]}$. 湖泊水位下降使得洞庭湖洲滩湿地提前出露, 淹没历时的减少又使得泥滩地上的植被提前萌发, 进而导致 湿地植被向湖心方向发生正向演替 ${ }^{[34-36]}$. 湿生植被群落结构和分布格局的变化会直接影响水鸟的食物来 源, 严重威胁鸟类赖以生存的栖息地. Zhao 等 ${ }^{[37]}$ 的研究表明, 洞庭湖湿地的白额雁 (Anser albifrons) 从 1980s 的 5 万只持续减少, 到 2010 年甚至不足 1000 只, 这主要是由于洞庭湖退水时间提前, 导致白额雁赖以生存 的苔草群落结构发生改变. 水文情势变化对水生生物的影响同样不能忽视, 水温、水质、水沙等条件随着水 文情势的变化而发生改变, 进而改变栖息地环境, 影响水生生物的生存、繁殖、新陈代谢等活动 ${ }^{[38-39]}$. 从 
ERHIs 来看, 年最小流量出现时间与鱼类群落的多样性有关, 低流量与鱼类的丰度有关 ${ }^{[18]}$. 朱轶等 ${ }^{[40]}$ 认为 三峡大坝汛末蓄水导致了西洞庭湖流量下降、高水位持续时间减少,使得鲤 (Cyprinus carpio)、鲫( Carassius auratus) 的生存空间持续减少, 是造成西洞庭湖鱼类多样性急剧下降的重要原因之一. 对于以流量变化为生 存策略的洄游产卵鱼类来说, 洪峰流量的削减会使这种信号减弱, 甚至消失, 从而影响洄游鱼类的生殖和繁 衍 $^{[41]}$. 洞庭湖出口的流量逆转次数呈现出明显的上升趋势 (图 4), 而流量逆转次数的变化则暗示着水生生 境也随之发生变化, 而这种变化的生境有可能更加适合外来人侵物种生存, 进而导致本地物种灭绝 ${ }^{[9]}$. 因 此,将洞庭湖的环境流量控制在合理的范围内将有利于缓解洞庭湖生态系统当前所面临的突出问题.

\section{3 结论}

1) 相关性分析揭示了 IHA 参数之间的高相关性, 说明其圥余性问题比较突出. PCA 的分析结果表明, 特征值大于 1 的前 8 个主成分的累积贡献率约为 $80 \%$. 据此确定的洞庭湖出口的 7 个 ERHIs 依次为年最大 90 日流量、年最小 3 日流量、年最小流量出现时间、3 月流量、6 月流量、流量逆转次数和低流量年内平均历 时. 通过与已有研究结果的横向对比以及 ERHIs 自身的纵向对比,都表明所选取的 7 个 ERHIs 是合理的.

2) ERHIs 能有效缓解 IHA 变量的圥余性问题,并保留了关键的生态水文信息. 根据 ERHIs 改进的 RVA 方法将原来的 32 个水文变量缩减至 17 个, 极大地简化了水资源调控目标. 该方法在洞庭湖出口环境流量 估算中的应用,显示了其具有更强的实用性,有利于通过较少的水文变量来描述生态系统对水文情势变化 的响应关系, 对水资源管理和生态系统保护都具有重要的参考价值和借鉴意义.

\section{4 参考文献}

[ 1 ] Huang YL, Fu BJ, Chen LD. Advances in ecohydrological process research. Acta Ecologica Sinica, 2003, 23(3) : 580587. [黄奕龙, 傅伯杰, 陈利顶. 生态水文过程研究进展. 生态学报, 2003, 23(3): 580-587.]

[ 2 ] Bunn SE, Arthington AH. Basic principles and ecological consequences of altered flow regimes for aquatic biodiversity. Environmental Management, 2002, 30(4) : 492-507. DOI: 10.1007/s00267-002-2737-0.

[ 3 ] Chang FJ, Tsai MJ, Tsai WP et al. Assessing the ecological hydrology of natural flow conditions in Taiwan. Journal of Hydrology, 2008, 354(1/2/3/4) : 75-89. DOI: 10.1016/j.jhydrol.2008.02.022.

[ 4 ] Zhang ZJ, Zhang Q, Gu XH et al. Changes of eco-flow due to hydrological alterations in the Yellow River Basin. Journal of Natural Resources, 2016, 31(12) : 2021-2033. DOI: 10.11849/zrzyxb.20160068. [张宗娇, 张强, 顾西辉等. 水文变 异条件下的黄河干流生态径流特征及生态效应. 自然资源学报, 2016, 31(12) : 2021-2033.]

[ 5 ] Yang ZF, Cui BS, Sun T et al eds. The mechanism, model and allocation for wetland ecological water requirement. Beijing: Science Press, 2012: 7-22. [杨志峰, 崔保山, 孙涛等. 湿地生态需水机理、模型和配置. 北京: 科学出版社, 2012: 7-22.]

[ 6 ] Richter BD, Baumgartner JV, Powell J et al. A method for assessing hydrologic alteration within ecosystems. Conservation Biology, 1996, 10(4) : 1163-1174. DOI: 10.1046/j.1523-1739.1996.10041163.x.

[ 7 ] The Nature Conservancy. Indicators of hydrologic alteration version 7.1 user's manual, 2009.

[ 8 ] Richter BD, Baumgartner JV, Wigington R et al. How much water does a river need? Freshwater Biology, 1997, 37( 1): 231-249. DOI: 10.1046/j.1365-2427.1997.00153.x.

[ 9 ] Suen JP. Potential impacts to freshwater ecosystems caused by flow regime alteration under changing climate conditions in Taiwan. Hydrobiologia , 2010, 649(1) : 115-128. DOI: 10.1007/s10750-010-0234-7.

[10] Zhang Q, Xiao M, Liu CL et al. Reservoir-induced hydrological alterations and environmental flow variation in the East River, the Pearl River basin, China. Stochastic Environmental Research and Risk Assessment, 2014, 28( 8 ) : 2119-2131. DOI: $10.1007 / \mathrm{s} 00477-014-0893-4$.

[11] Jiang L, Ban X, Wang X et al. Assessment of hydrologic alterations caused by the Three Gorges Dam in the middle and lower reaches of Yangtze River, China. Water, 2014, 6(5) : 1419-1434. DOI: 10.3390/w6051419.

[12] Mathews R, Richter BD. Application of the indicators of hydrologic alteration software in environmental flow setting. Journal of the American Water Resources Association, 2007, 43(6) : 1400-1413. DOI: 10.1111/j.1752-1688.2007.00099.x.

[13] Lin K, Lian Y, Chen X et al. Changes in runoff and eco-flow in the Dongjiang River of the Pearl River Basin, China. 
Frontiers of Earth Science, 2014, 8(4) : 547-557. DOI: 10.1007/s11707-014-0434-y.

[14] Yang Z, Yan Y, Liu Q. Assessment of the flow regime alterations in the Lower Yellow River, China. Ecological Informatics, 2012, 10: 56-64. DOI: 10.1016/j.ecoinf.2011.10.002.

[15] Olden JD, Poff NL. Redundancy and the choice of hydrologic indices for characterizing streamflow regimes. River Research and Applications, 2003, 19(2) : 101-121. DOI: 10.1002/rra.700.

[16] Clausen B, Biggs BJF. Flow variables for ecological studies in temperate streams: groupings based on covariance. Journal of Hydrology, 2000, 237(3/4) : 184-197. DOI: 10.1016/s0022-1694(00) 00306-1.

[17] Smakhtin VU, Shilpakar RL, Hughes DA. Hydrology-based assessment of environmental flows: an example from Nepal. Hydrological Sciences Journal, 2006, 51(2) : 207-222. DOI: 10.1623/hysj.51.2.207.

[18] Yang YCE, Cai X, Herricks EE. Identification of hydrologic indicators related to fish diversity and abundance: A data mining approach for fish community analysis. Water Resources Research, 2008, 44 ( 4): W04412. DOI: 10. 1029/2006WR005764.

[19] Gao Y, Vogel RM, Kroll CN et al. Development of representative indicators of hydrologic alteration. Journal of Hydrology, 2009, 374(1/2) : 136-147. DOI: 10.1016/j.jhydrol.2009.06.009.

[20] Gu XH, Zhang Q, Kong DD et al. Based on multiple hydrological alteration indicators evaluating the characteristics of flow regime with the impact on the diversity of hydrophily biology. Acta Ecologica Sinica, 2016, 36(19) : 6079-6090. DOI: 10. 5846/stxb201412122480. [顾西辉, 张强, 孔冬冬等. 基于多水文改变指标评价东江流域河流流态变化及其对生物 多样性的影响. 生态学报, 2016, 36(19) : 6079-6090.]

[21] Yang B, He BH, Wang DB. Hanfeng Pre-reservoir commissioning time variation feature of the hydrology and water quality in Three Gorges Reservoir. Environmental Science, 2017, 38(4) : 1366-1375. DOI : 10.13227/j.hjkx.201607173. [杨兵, 何丙辉, 王德宝. 三峡前置库汉丰湖试运行年水文水质变化特征. 环境科学, 2017, 38(4) : 1366-1375. ]

[22] Arabzadeh R, Kholoosi MM, Bazrafshan J. Regional hydrological drought monitoring using principal components analysis. Journal of Irrigation and Drainage Engineering, 2016, 142(1) : 04015029. DOI: 10.1061/(ASCE) IR.1943-4774.0000925.

[23] Olsen RL, Chappell RW, Loftis JC. Water quality sample collection, data treatment and results presentation for principal components analysis-literature review and Illinois River watershed case study. Water Research, 2012, 46 (9) : 31103122. DOI: 10.1016/j.watres.2012.03.028.

[24] Yu H, Wu ZR, Bao TF et al. Multivariate analysis in dam monitoring data with PCA. Scientia Sinica Technologica, 2010, 40(7) : 830-839. DOI: 10.1007/s11431-010-0060-1. [虞鸿, 吴中如, 包腾飞等. 基于主成分的大坝观测数据多效 应量统计分析研究. 中国科学: 技术科学, 2010, 40(7):830-839.]

[25] Turner M, Stewardson M. Hydrologic indicators of hydraulic conditions that drive flow-biota relationships. Hydrological Sciences Journal-Journal Des Sciences Hydrologiques, 2014, 59(3/4) : 659-672. DOI: 10.1080/02626667.2014.896997.

[26] Poff NL, Allan JD, Bain MB et al. The natural flow regime. Bioscience, 1997, 47 ( 11): 769-784. DOI: 10. $2307 / 1313099$.

[27] Zhang Q, Gu X, Singh VP et al. Evaluation of ecological instream flow using multiple ecological indicators with consideration of hydrological alterations. Journal of Hydrology, 2015, 529 (Part 3): 711-722. DOI: 10. 1016/j. jhydrol. 2015. 08.066 .

[28] Cui BS, Zhao X, Yang ZF. Eco-hydrology-based calculation of the minimum ecological water requirement for lakes. Acta Ecologica Sinica, 2005, 25(7) : 1788-1795. [崔保山, 赵翔, 杨志峰. 基于生态水文学原理的湖泊最小生态需水量 计算. 生态学报, 2005, 25(7): 1788-1795.]

[29] Lai X, Liang Q, Jiang J et al. Impoundment effects of the Three-Gorges-Dam on flow regimes in two China's largest freshwater lakes. Water Resources Management, 2014, 28(14) : 5111-5124. DOI: 10.1007/s11269-014-0797-6.

[30] Huang Q, Sun Z, Opp C et al. Hydrological drought at Dongting Lake: Its detection, characterization, and challenges associated with Three Gorges Dam in central Yangtze, China. Water Resources Management, 2014, 28 ( 15 ) : 5377-5388. DOI: $10.1007 / \mathrm{s} 11269-014-0807-8$.

[31] Qin HY, Xie YH, Zou DS. Succession trend, reason analysis, prevention and reduction measures of agriculture flood and drought disasters in Hunan province. Research of Agricultural Modernization, 2011, 32(2) : 165-169. [覃红燕, 谢永宏, 邹冬生. 湖南省农业洪早灾害演变趋势和成因及防灾减灾措施. 农业现代化研究, 2011, 32(2) : 165-169.]

[32] Yang G, Zhang Q, Wan R et al. Lake hydrology, water quality and ecology impacts of altered river-lake interactions: ad- 
vances in research on the middle Yangtze river. Hydrology Research, 2016, 47(S1) : 1-7. DOI: 10.2166/nh.2016.003.

[33] Wu H, Zeng G, Liang J et al. Responses of landscape pattern of China's two largest freshwater lakes to early dry season after the impoundment of Three-Gorges Dam. International Journal of Applied Earth Observation and Geoinformation, 2017, 56: 36-43. DOI: 10.1016/j.jag.2016.11.006.

[34] Xie YH, Tang Y, Chen XS et al. The impact of Three Gorges Dam on the downstream eco-hydrological environment and vegetation distribution of East Dongting Lake. Ecohydrology, 2015, 8(4) : 738-746. DOI: 10.1002/eco.1543.

[35] Hu YX, Huang JL, Du Y et al. Monitoring wetland vegetation pattern response to water-level change resulting from the Three Gorges Project in the two largest freshwater lakes of China. Ecological Engineering, 2015, 74: 274-285. DOI: 10. 1016/j.ecoleng.2014.10.002.

[36] Lai XJ, Jiang JH, Huang Q. Pattern of impoundment effects and influencing mechanism of Three Gorges Project on water regime of Lake Dongting. J Lake Sci, 2012, 24(2) : 178-184. DOI:10.18307/2012.0202. [赖锡军, 姜加虎, 黄群. 三 峡工程蓄水对洞庭湖水情的影响格局及其作用机制. 湖泊科学, 2012, 24(2): 178-184.]

[37] Zhao M, Cong P, Barter M et al. The changing abundance and distribution of greater white-fronted geese Anser albifrons in the Yangtze River floodplain: impacts of recent hydrological changes. Bird Conservation International, 2012, 22(2) : 135143. DOI: $10.1017 / \mathrm{s} 0959270911000542$.

[38] Li J, Xia ZQ, Dai HC et al. Effect of the Three Gorges Reservoir initial filling on downstream habitat suitability of the typical fishes. Journal of Hydraulic Engineering, 2013, 44(8): 892-900. [李建, 夏自强, 戴会超等. 三峡初期蓄水对典 型鱼类栖息地适宜性的影响. 水利学报, 2013, 44(8):892-900.]

[39] Gooseff MN, Wlostowski A, McKnight DM et al. Hydrologic connectivity and implications for ecosystem processes - Lessons from naked watersheds. Geomorphology, 2017, 277: 63-71. DOI: 10.1016/j.geomorph.2016.04.024.

[40] Zhu Y, Lu C, Hu HJ et al. Changes in fish community structure in West Dongting Lake after the operation of the Three Gorges Dam. J Lake Sci, 2014, 26(6) : 844-852. DOI: 10.18307/2014.0605. [ 朱轶, 吕偲, 胡慧建等. 三峡大坝运行 前后西洞庭湖鱼类群落结构特征变化. 湖泊科学, 2014, 26(6) : 844-852.]

[41] Lytle DA, Poff NL. Adaptation to natural flow regimes. Trends in Ecology \& Evolution, 2004, 19(2) : 94-100. DOI: 10. 1016/j.tree.2003.10.002. 